\title{
CONFORMALLY FLAT MANIFOLDS WHOSE DEVELOPMENT MAPS ARE NOT SURJECTIVE. I
}

BY

\author{
YOSHINOBU KAMISHIMA
}

\begin{abstract}
Let $M$ be an $n$-dimensional conformally flat manifold. A universal covering of $M, \tilde{M}$ admits a conformal development map into $S^{n}$. When a development map is not surjective, we can relate the boundary of the development image with the limit set of the holonomy group of $M$. In this paper, we study properties of closed conformally flat manifolds whose development maps are not surjective.
\end{abstract}

Introduction. A conformally flat manifold is a Riemannian manifold whose Riemannian metric is locally conformally equivalent to the euclidean metric on $\mathbf{R}^{n}$. We study the discreteness of holonomy groups of closed conformally flat manifolds whose development maps are not surjective. Our main result is

THEOREM A. Let $M$ be a 3-dimensional closed conformally flat manifold whose development map is not surjective. Then either the holonomy group is discrete, or $M$ is a locally homogeneous Riemannian manifold of nonpositive sectional curvature (in which case some finite covering of $M$ is diffeomorphic to the product $S^{1} \times N_{g}$, where $N_{g}$ is a compact surface of genus $g \geqslant 2$ ).

THEOREM B. Let $M$ be an $n(\geqslant 3)$-dimensional closed conformally flat manifold. If the development map is not surjective, then it is a covering map.

When the holonomy group is solvable, we have the following result related to the work of W. Goldman [6].

THEOREM C. Let $M$ be an $n$ ( $\geqslant 3$-dimensional closed conformally flat manifold. If the holonomy group is virtually solvable, then $M$ is a spherical space form, a euclidean space form, or a nonnegatively curved manifold finitely covered by a Hopf manifold $S^{1} \times S^{n-1}$.

The holonomy group of an $n$-dimensional conformally flat manifold belongs to the group of conformal transformations of the $n$-sphere $S^{n}$. It also acts as the group of isometries of $(n+1)$-dimensional hyperbolic space, and thus the limit set is defined for the holonomy group. When the development map is not surjective, we can relate the boundary of the development image with this limit set. In order to study the discreteness of holonomy groups, we assume that the holonomy group $\Gamma$ sits inside a positive dimensional closed subgroup $G$ as the group of isometries with

Received by the editors March 4, 1985 and, in revised form, May 27, 1985.

1980 Mathematics Subject Classification. Primary 57S30, 57R55, 57 S17.

(C)1986 American Mathematical Society $0002-9947 / 86 \$ 1.00+\$ .25$ per page 
the limit sets $L(\Gamma)=L(G)$. Under this assumption, if $G$ has finitely many components, then such conformally flat manifolds are easily classified. (See Theorem 3.1 and Corollary 3.4.) In fact, these manifolds are locally homogeneous Riemannian manifolds (cf. Kuiper [11], Takagi [22]).

Further, if the holonomy group is discrete, then it is proved that the notion of limit sets in $S^{n}$ for a group of conformal transformations (cf. [12]) coincides with that of the limit set as above. In our case, the boundary of the development image is shown to be equal to the limit set. Thus the classification of conformally flat manifolds whose development maps are not surjective and with discrete holonomy groups is closely related to the study of dynamical properties of groups of conformal transformations on higher-dimensional spheres. The classification of limit sets of Kleinian groups is far from complete, even in the 2-dimensional case (cf. [23, 24]). Our assumption on the limit sets will impose a strong restriction on holonomy groups. It will be seen that the limit set lies in the boundary of a totally geodesic submanifold in a hyperbolic space.

The necessary facts of conformal and hyperbolic geometries will be explained in $\S 1$ as well as the definition of a conformally flat manifold. In $\S 2$, we shall study the limit sets mentioned above, and $\S 3$ will be devoted to the proofs of statements.

I would like to thank Professor W. Goldman who gave a suggestion for Theorem C. He also showed me an example of a closed conformally flat irreducible 3-manifold with a surjective development map [26, §5]. I would also like to thank M. Feighn for reading my draft carefully. Finally, my motivation is due to the notion of visibility manifolds as developed by Bishop, O’Neill, Chen and Eberlein [1-4] and also Kulkarni's work concerning the uniformization principle and the limit set [12, 13]. I gratefully acknowledge the hospitality of Professor Ravi S. Kulkarni and Indiana University during my short visit.

1. Preliminaries and example of locally homogeneous conformally flat manifolds. Let $G$ be a subgroup of $\operatorname{GL}(n, \mathbf{R})$. A $G$-structure of an $n$-dimensional smooth manifold $M$ is a reduction of the structure group from $\operatorname{GL}(n, \mathbf{R})$ to $G$ of the associated principal bundle $L(M)$ to the tangent bundle of $M$. Equivalently, a $G$-structure on $M$ is a principal $G$-subbundle of $L(M)$. A $G$-structure on $M$ is said to be integrable if there exists a coordinate neighborhood of $p$ for each $p \in M$ with coordinates $\left(x_{1}, \ldots, x_{n}\right)$ and the coordinate frame $\left(\partial / \partial x_{1}, \ldots, \partial / \partial x_{n}\right)$ belongs to this subbundle. Suppose that $G=\mathrm{O}(n) \times \mathbf{R}^{+}$, consisting of matrices $A$ with $A^{t} A=$ $a I$ for $a>0$. It is easy to see that the conformal equivalence classes of Riemannian metrics on $M$ is naturally in a one-to-one correspondence with $G$-structures on $M$. Furthermore, a $G$-structure is integrable if and only if any Riemannian metric corresponding to the $G$-structure is locally conformally equivalent to the flat euclidean metric on $\mathbf{R}^{n}$. (For details see $[9,13]$.) A conformally flat manifold is a Riemannian manifold with an integrable $G$-structure, or equivalently a Riemannian manifold whose Riemannian metric is locally conformally equivalent to the euclidean metric on $\mathbf{R}^{n}$.

Let $S^{n}$ be the quadric hypersurface in the projective space $P^{n+1}$ defined by 
$\left\{\left[x_{0}, \ldots, x_{n+1}\right] \in P^{n+1} \mid-x_{0}^{2}+x_{1}^{2}+\cdots+x_{n+1}^{2}=0\right\}$. Then $S^{n}$ is isometric to the standard sphere of constant positive curvature (cf. [9]). By stereographic projection, $S^{n}$ is conformally flat and thus it admits an integrable $G$-structure. $\operatorname{Let} \operatorname{SO}(1, n+1)$ denote the subgroup of the Lorentz group $\mathrm{O}(1, n+1)$ preserving $S^{n}$. It is a theorem of Liouville that the group of conformal transformations of $S^{n}, \operatorname{Conf}\left(S^{n}\right)$, is $\mathrm{SO}(1, n+1) . \mathrm{SO}(1, n+1)$ is transitive on $S^{n}$, and the stabilizer at $e=[1,0, \ldots, 0,1]$ is called the similarity transformations of $\mathbf{R}^{n}$. Here, $\mathbf{R}^{n}$ is identified with $S^{n}-\{e\}$. Note that the group of similarity transformations, $\operatorname{Sim}\left(\mathbf{R}^{n}\right)$, is isomorphic to $\mathbf{R}^{n} \rtimes$ $\left(\mathrm{O}(n) \times \mathbf{R}^{+}\right)$. Let $S_{e}^{n}$ denote the tangent space of $S^{n}$ at $e$. The image $\tau\left(\operatorname{Sim}\left(\mathbf{R}^{n}\right)\right)$ of the isotropy representation $\tau: \operatorname{Sim}\left(\mathbf{R}^{n}\right) \rightarrow S_{e}^{n}$ is $G=\mathrm{O}(n) \times \mathbf{R}^{+}$. Then it follows from a fundamental result of Kulkarni [13, Proposition 2.1] that any manifold uniformized smoothly over $S^{n}$ with respect to $\mathrm{SO}(1, n+1)$ carries an integrable $G$-structure; i.e., it admits a conformally flat structure. The converse is also true, provided that $n>2$, as proved by Kuiper [10] and Kulkarni [12]. Consequently, we have the following uniformization theorem.

TheOREM $1.1[6,10,13]$. Suppose $n \geqslant 3$. Let $M^{n}=\pi \backslash X$ be a smooth $n$-dimensional manifold, where $\pi$ is the fundamental group of $M$ and $X$ is a universal covering of $M$. Then $M$ is a conformally flat manifold if and only if it is uniformizable over $S^{n}$ with respect to $\operatorname{Conf}\left(S^{n}\right)$. In that case, there exists a conformal immersion $d: X \rightarrow S^{n}$ and a homomorphism $\phi: \pi \rightarrow \operatorname{Conf}\left(S^{n}\right)$ such that the following diagram is commutative for $\gamma \in \pi$ :

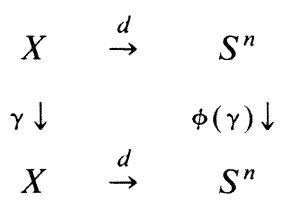

Furthermore, such a pair $(d, \phi)$ is unique up to conjugation by an element of $\operatorname{Conf}\left(S^{n}\right)$.

The map $d$ is called a development map, and $\phi$ is called the holonomy homomorphism of $\pi . \phi(\pi)$ is said to be the holonomy group of $\pi$. We will use these notations throughout this paper unless otherwise mentioned. On the other hand, from the point of view of classical automorphic function theory we can interpret conformal geometry in terms of the notions of visibility manifolds (cf. [2-4]) as follows. Let $Y$ be a complete simply connected Riemannian manifold of nonpositive sectional curvature. An equivalence class of asymptotic geodesics is a point at infinity, and the set of all points at infinity is denoted by $\partial Y$. The space $\bar{Y}=Y \cup \partial Y$ together with the cone topology is a compactification of $Y$. The space $\bar{Y}$ is homeomorphic to the closed ball of $\operatorname{dim} Y$. We call $\partial Y$ the boundary of $Y$. Let $I(Y)$ be the group of isometries of $Y$. A subgroup $\Gamma$ of $I(Y)$ determines a limit set $L(\Gamma) \subset \partial Y$, which is the set of cluster points of an orbit $\Gamma p$ for $p \in Y$. It is noted that $L(\Gamma)$ does not depend on the choice of a point $p$ in $Y$. Each element of $I(Y)$ extends naturally to a 
homeomorphism of $\bar{Y}$ onto itself. $Y$ is called a visibility manifold if, for distinct $x, y$ in $\partial Y$, there exists a geodesic joining $x$ and $y$.

Now, the typical example of visibility manifolds is a complete simply connected Riemannian manifold of constant negative curvature -1 (i.e., a hyperbolic space $\left.\mathbf{H}^{n+1}\right)$. The boundary of $\mathbf{H}^{n+1}$ is the standard sphere $S^{n} . I\left(\mathbf{H}^{n+1}\right)$ is the Lorentz group $\mathrm{SO}(1, n+1)$.

It is well known that the geometry of $\left(\mathrm{SO}(1, n+1), \mathbf{H}^{n+1}\right)$ limits the boundary as $\left(\operatorname{Conf}\left(S^{n}\right), S^{n}\right)$. Following this reinterpretation, the aim of this paper is to study conformal actions of $S^{n}$ in connection with isometric actions of $\mathbf{H}^{n+1}$. More general results concerning visibility manifolds can be found in Chen, Greenberg, Eberlein and O'Neill's works.

We quote two necessary facts about hyperbolic spaces from those of [2-4]. Points $x, y$ in $\partial Y$ are said to be dual relative to a subgroup $\Gamma$ of $I(Y)$ if there exists a sequence $\left\{\gamma_{n}\right\} \subset \Gamma$ such that $\gamma_{n} p \rightarrow y, \gamma_{n}^{-1} p \rightarrow x$ for every $p \in Y$. The following first fact is due to Eberlein and O'Neill (cf. [4, Definition 8.2, Propositions 8.3, 8.4; 2]).

Proposition 1.2. Let $Y$ be a visibility manifold. If $x$ and $y$ (not necessarily distinct) are dual relative to a subgroup $\Gamma$ of $I(Y)$, then given any neighborhood $U, V$ of $x$, $y$, respectively, in $\partial Y$ there exists $\gamma \in \Gamma$ such that $\gamma(\partial Y-U) \subseteq V$ (and hence $\left.\gamma^{-1}(\partial Y-V) \subseteq U\right)$. Furthermore, if $\left\{\gamma_{n}\right\}$ is as in the definition, then $\gamma$ can be taken to be $\gamma_{n}$ for sufficiently large $n$.

When we restrict our manifolds to the hyperbolic space $\mathbf{H}^{n+1}$, we have the following useful lemma, obtained by Chen and Greenberg [3, Lemma 4.3.3, 27, Chapter 8].

LEMma 1.3. Let $C$ be a closed subset of $S^{n}$ which contains more than one point and is invariant under a subgroup $\Gamma$ of $I\left(\mathbf{H}^{n+1}\right)$. Then $L(\Gamma) \subset C$.

Let $D$ be the set of points in $\partial Y$ dual to $x \in \partial Y$. Then it is easy to see that $D$ is a closed $\Gamma$-invariant subset of $L(\Gamma)$. If $Y=\mathbf{H}^{n+1}$ and $D$ contains more than one point, we have

$$
D=L(\Gamma) .
$$

1.5. Examples of locally homogeneous conformally flat manifolds. Let $S^{n}$ be the quadric hypersurface in $P^{n+1} . S^{k}$ is defined to be the subset of $S^{n},\left\{\left[x_{0}, \ldots, x_{n+1}\right]\right.$ $\left.\in S^{n} \mid x_{k+2}=\cdots=x_{n+1}=0\right\}$ for $0 \leqslant k \leqslant n-1 . S^{k}$ is the standard $k$-dimensional sphere via the correspondence $x \rightarrow\left(x_{1} / x_{0}, \ldots, x_{k+1} / x_{0}\right)$.

LEMMA 1.6. For $0 \leqslant k \leqslant n-1$, there exists an equivariant diffeomorphism of $S^{n}-S^{k}$ onto $\mathbf{H}^{k+1} \times S^{n-(k+1)}$ with respect to the group of conformal transformations of $S^{n}$ preserving $S^{n}-S^{k}$ and the group $\mathrm{SO}(1, k+1) \times 0(n-k)$.

Proof. For $x=\left[x_{0}, \ldots, x_{n+1}\right] \in S^{n}-S^{k}$, we may assume $x_{0}>0$. If we put $a$ $=\sqrt{x_{k+2}^{2}+\cdots+x_{n+1}^{2}}$, then the correspondence $f$ defined by

$$
f(x)=\left(\left(x_{0} / a, \ldots, x_{k+1} / a\right),\left(x_{k+2} / a, \ldots, x_{n+1} / a\right)\right)
$$


is a diffeomorphism of $S^{n}-S^{k}$ onto $\mathbf{H}^{k+1} \times S^{n-(k+1)}$. Furthermore, under this identification, it is easy to see that the group of conformal transformations preserving $S^{n}-S^{k}$ is actually isomorphic to $\mathrm{SO}(1, k+1) \times \mathrm{O}(n-k)$ (cf. [3, Lemma 4.2.1]).

1.7. Suppose $n \geqslant 3$. If we choose a complete Riemannian metric of constant curvature -1 (resp. constant curvature +1 ) on $\mathbf{H}^{k+1}$ (resp. $S^{n-(k+1)}$ ), then the product metric defines a complete Riemannian metric on $\mathbf{H}^{k+1} \times S^{n-(k+1)}$ for which the group of isometries is $\mathrm{SO}(1, k+1) \times \mathrm{O}(n-k)$. Of course, when $k=0$ or $n-2$, choose the usual flat metric on $\mathbf{H}^{1}$ or $S^{1}$. Thus, $\mathbf{H}^{n-1} \times S^{1}$ is a complete Riemannian manifold of nonpositive sectional curvature. Similarly, $\mathbf{H}^{1} \times S^{n-1}$ is one of nonnegative sectional curvature. If $\Gamma$ is a torsion free discrete subgroup of $\mathrm{SO}(1, k+1) \times \mathrm{O}(n-k)$, then $\Gamma \backslash\left(\mathbf{H}^{k+1} \times S^{n-(k+1)}\right)$ is a locally homogeneous Riemannian manifold and $f$ induces an isometry of $\Gamma \backslash\left(\mathbf{H}^{k+1} \times S^{n-(k+1)}\right)$ onto $f^{-1} \Gamma f \backslash S^{n}-S^{k}$ relative to the induced metric. Since $f^{-1} \Gamma f$ is a subgroup of $\operatorname{Conf}\left(S^{n}\right)$ that acts properly discontinuously and freely on $S^{n}-S^{k}$, the orbit space is obviously uniformizable over $S^{n}$ with respect to $\operatorname{Conf}\left(S^{n}\right)$ and hence so is $\Gamma \backslash\left(\mathbf{H}^{k+1} \times S^{n-(k+1)}\right)$. Then it follows from Theorem 1.1 that

$$
\Gamma \backslash\left(\mathbf{H}^{k+1} \times S^{n-(k+1)}\right)
$$

is a conformally flat manifold whose development map is chosen to be the conformal embedding $f^{-1}$.

(1) $1 \leqslant k \leqslant n-2$. Choose a torsion free discrete uniform subgroup $\Gamma$ of

$$
\mathrm{SO}(1, k+1) \times \mathrm{O}(n-k)
$$

Then $\Gamma \backslash\left(\mathbf{H}^{k+1} \times S^{n-(k+1)}\right)$ is a closed conformally flat manifold whose holonomy group is discrete and isomorphic to $\Gamma$. In the case that $k=n-2, \Gamma \backslash\left(\mathbf{H}^{n-1} \times S^{1}\right)$ is a compact Riemannian manifold of nonpositive sectional curvature.

(2) $k=0$. Choose a discrete infinite subgroup $\Gamma$ of $\mathrm{SO}(1,1) \times \mathrm{O}(n)$ which acts freely on $\mathbf{H}^{1} \times S^{n-1}$ (note that $\mathrm{SO}(1,1)^{0} \cong \mathbf{R}^{1}$ ). Then $\Gamma \backslash\left(\mathbf{H}^{1} \times S^{n-1}\right.$ ) is a compact Riemannian manifold of nonnegative sectional curvature. In this case, $\Gamma$ is isomorphic to either a group $F \rtimes \mathbf{Z}$ or an extension of $F \rtimes \mathbf{Z}$ by $\mathbf{Z}_{2}$ for some finite subgroup $F \subset \mathrm{O}(n)$. (See Corollary 2.4; also [5, 28, §2].) $\Gamma \backslash\left(\mathbf{H}^{1} \times S^{n-1}\right)$ is finitely covered by a Hopf manifold $S^{1} \times S^{n-1}$.

(3) $k=n-1$. Choose a torsion free discrete uniform subgroup $\Gamma$ of $\operatorname{SO}(1, n)$. For a connected component $\mathbf{H}^{n}$ of $\mathbf{H}^{n} \times S^{0}, \Gamma \backslash \mathbf{H}^{n}$ is a hyperbolic space form. In summary, we have

Proposition 1.8. Suppose $n \geqslant 3$. For each torsion free discrete uniform subgroup $\Gamma$ of $\mathrm{SO}(1, k+1)(0 \leqslant k \leqslant n-1)$ there exists an $n$-dimensional locally homogeneous conformally flat manifold such that (1) the development map is not surjective, and (2) the holonomy group is discrete and isomorphic to $\Gamma$. 
2. The limit sets of $S^{n}$. We consider the following sets: let $\Gamma$ be a subgroup of $\operatorname{Conf}\left(S^{n}\right)$. Then

$\Lambda_{0}=$ the closure of the set of points $x \in S^{n}$ with isotropy subgroup $\Gamma_{x}$ infinite.

$\Lambda_{1}=$ the set of cluster points of $\{\gamma z \mid \gamma \in \Gamma\}$, where $z \in S^{n}-\Lambda_{0}$.

$\Lambda_{2}=$ the set of cluster points of $\{\gamma K \mid \gamma \in \Gamma\}$,

where $K$ is a compact subset of $S^{n}-\left\{\Lambda_{0} \cup \Lambda_{1}\right\}$.

Following the notation of Kulkarni [12], $\Lambda=\Lambda_{0} \cup \Lambda_{1} \cup \Lambda_{2}$ is called the limit set of $\Gamma$. The set $\Omega=S^{n}-\Lambda$ is called the domain of discontinuity for $\Gamma$. It is a result of Kulkarni [12] that if $\Omega \neq \varnothing$, then $\Gamma$ acts properly discontinuously on $\Omega$, and thus $\Gamma$ is discrete in $\operatorname{Conf}\left(S^{n}\right)$. Recall that there is another limit set $L(\Gamma)$. In the definition of the limit set in the classical case of Kleinian groups of Möbius transformations on a Riemannian sphere, a special feature of conformal geometry is implicitly used. One might expect that the above notion of the limit sets coincides with the classical one in the higher-dimensional conformal geometry.

Proposition 2.1. Let $\Gamma$ be a discrete subgroup of $\operatorname{Conf}\left(S^{n}\right)$. If $\Lambda_{0} \neq S^{n}$, then $\Lambda=\Lambda_{0}=\Lambda_{1}=\Lambda_{2}=L(\Gamma)$.

REMARK. The definition of limit sets does not make sense for finite groups. So we may assume that $\Gamma$ is not a finite group. Then in our case $\Gamma$ is not a torsion group. This follows from a result of Tits that every discrete subgroup of $\operatorname{GL}(n, \mathbf{R})$ is either virtually polycyclic or contains a noncyclic free group $\mathbf{Z} * \mathbf{Z}$, and thus $\Gamma$ contains an element of infinite order.

Proof of Proposition 2.1. Since $\Gamma$ is discrete in $\operatorname{SO}(1, n+1)=\operatorname{Conf}\left(S^{n}\right)$, it acts properly discontinuously on $\mathbf{H}^{n+1}$. In particular, every element of infinite order is a nonelliptic element.

Step 1. Suppose that $\Gamma$ has a fixed point. Then we will prove that $\Lambda=\Lambda_{0}=\Lambda_{1}$ $=\Lambda_{2}=L(\Gamma)=$ one point $\}$, and $\Gamma$ is a discrete subgroup of $E(n)$; or $\Lambda=\Lambda_{0}=$ $\Lambda_{1}=\Lambda_{2}=L(\Gamma)=$ two points $\}$, and $\Gamma$ is a discrete subgroup of $\mathrm{O}(n) \times \mathbf{R}^{+}$ isomorphic to $F \rtimes \mathbf{Z}$ for some finite subgroup $F \subset \mathrm{O}(n)$. If $\Gamma$ has a fixed point, then $\Gamma$ is a discrete subgroup of $\operatorname{Sim}\left(\mathbf{R}^{n}\right)=\mathbf{R}^{n} \rtimes\left(\mathrm{O}(n) \times \mathbf{R}^{+}\right)$. If $\Gamma$ does not intersect with $E(n)=\mathbf{R}^{n} \rtimes \mathrm{O}(n)$, then it is isomorphic to its image in $\mathbf{R}^{+}$. Since every discrete subgroup of $\operatorname{Sim}\left(\mathbf{R}^{n}\right)$ is finitely generated (i.e., virtually polycyclic), $\Gamma$ is a free abelian subgroup of finite rank. In general, the limit set $L(A)$ for an abelian group $A$ consisting of nonelliptic elements consists of at most two points and is the set of fixed points of $A$. If $\Gamma$ is as above, then $L(\Gamma)$ consists of exactly two points, and hence $\Gamma$ is isomorphic to $\mathbf{Z}$. Further, consider the real algebraic closure of $\Gamma$, $A(\Gamma)$, in $\operatorname{Sim}\left(\mathbf{R}^{n}\right)$. Then $A(\Gamma)$ is abelian, and hence $A(\Gamma)=K \times \mathbf{R}^{+}$for some compact abelian group $K \subset \mathrm{O}(n)$. If the intersection with $E(n)$ is nontrivial, then it follows as in the argument of [8, Proposition 2.6] that $\Gamma \subset \mathrm{O}(n) \times \mathbf{R}^{+}$or $\Gamma \subset E(n)$. If $\Gamma \subset \mathrm{O}(n) \times \mathbf{R}^{+}$, then it is easy to see that $\Gamma=F \rtimes \mathbf{Z}$ for some finite group $F \subset \mathrm{O}(n)$, and $L(\Gamma)$ consists of two points. If $\Gamma \subset E(n)$, then $\Gamma$ has a free abelian 
subgroup $A$ of finite index. We have $L(\Gamma)=L(A)$ and $L(A)$ consists of a unique fixed point. Next we prove the equality for the limit sets. Let $a$ be an arbitrary point in $\Lambda_{0}$. Then there exists $\gamma \in \Gamma$ such that $\gamma a=a$. If $x \neq a$, then there exists a unique geodesic $\alpha$ joining $a$ and $x$ which is translated by the nonelliptic element $\gamma$ (cf. [4, Proposition 6.4]). Then $a$ is either $\alpha(\infty)=\lim _{i \rightarrow \infty} \gamma^{i} \alpha(0)$ or $\alpha(-\infty)=$ $\lim _{i \rightarrow \infty} \gamma^{-i} \alpha(0)$. It follows that $a \in L(\Gamma)$, and thus $\Lambda_{0}=L(\Gamma)$ by the definition of $\Lambda_{0}$. If $a$ is an arbitrary point in $\Lambda_{1}$, then there exist a sequence $\left\{\gamma_{i}\right\} \subset \Gamma$ and $z \in S^{n}-\Lambda_{0}$ such that $a=\lim _{i \rightarrow \infty} \gamma_{i} z$. Consider the dual points $b=\lim \gamma_{i} p$ and $b^{\prime}=\lim \gamma_{i}^{-1} p\left(p \in \mathbf{H}^{n+1}\right)$. Note that these points lie in $L(\Gamma)$; otherwise $\Gamma$ has a limit point in $\mathbf{H}^{n+1}$ and so fails to be properly discontinuous. Choose $a$ neighborhood $U$ of $b^{\prime}$ in $S^{n}$ which does not contain $z$. Then for an arbitrary neighborhood $V$ of $b$, it follows from Proposition 1.2 that $\gamma_{n}\left(S^{n}-U\right) \subset V$ for sufficiently large $n$; i.e., $\gamma_{n} z \in V$. We obtain $a=\lim \gamma_{n} z=b \in L(\Gamma)$, so that $\Lambda_{1} \subset L(\Gamma)$. If $a$ is as above, then $a^{\prime}=\lim \gamma_{i}^{-1} z \in \Lambda_{1}$, and the same argument is applied to show that $a^{\prime}=b^{\prime}$. According to the structure of $L(\Gamma)$, it follows that either $b=b^{\prime}=x$ or $b \neq b^{\prime}$ and $\left\{b, b^{\prime}\right\}=\{x, y\}$. And hence, $\Lambda_{1}=L(\Gamma)$. Let $a$ be an arbitrary point in $\Lambda_{2}$. Then there exist a sequence $\left\{\gamma_{i}\right\} \subset \Gamma$ and a compact set $K \subset S^{n}-\left\{\Lambda_{0} \cup \Lambda_{1}\right\}$ for which every neighborhood of $a$ intersects with some $\gamma_{i} K$. Note that $K \cap L(\Gamma)$ $=\varnothing$. When we consider the dual points $b=\lim \gamma_{i} p, b^{\prime}=\lim \gamma_{i}^{-1} p$ again, it follows similarly that for an arbitrary neighborhood $V$ of $b, \gamma_{n} K \subset V$. And thus we obtain $a=b$. If we note that the sequence $\left\{\gamma_{i}^{-1} K\right\}$ has an accumulation point $a^{\prime}$ in $\Lambda_{2}$, it follows that $a^{\prime}=b^{\prime}$. And hence $\Lambda_{2}=L(\Gamma)$. This proves Step 1 .

Step 2. Suppose that $\Gamma$ has no fixed points. Then each $\Lambda_{i}$ contains more than one point since they are closed $\Gamma$-invariant subsets. It follows from Lemma 1.3 that $L(\Gamma) \subset \Lambda_{i}(i=0,1,2)$. If $a$ is a point in $\Lambda_{0}$, then $\gamma a=a$ for some $\gamma \in \Gamma$ as before. Consider the point $x=\lim _{i \rightarrow \infty} \gamma^{i} p \in L(\Gamma)\left(p \in \mathbf{H}^{n+1}\right) . \gamma$ fixes $a$ and $x$. If $a \neq x$, then as in the argument of Step 1, we have $a \in L(\Gamma)$, so that $L(\Gamma)=\Lambda_{0}$. When we consider again dual points for $\Lambda_{1}$ and $\Lambda_{2}$, respectively, the same argument of Step 1 shows that $\Lambda_{i} \subset L(\Gamma)$. And hence $\Lambda_{i}=L(\Gamma)(i=1,2)$. It is noted that in this case there are in general no relations between the dual points.

REMARK 2.2. If $\Gamma$ is not discrete in $\operatorname{Conf}\left(S^{n}\right)$ (this is the case that $\Omega=\varnothing$ ), then the above equality obviously fails. For example, take an infinite cyclic subgroup $\mathbf{Z}$ in $\mathrm{SO}(2)$ where $\mathrm{SO}(2) \subset \mathrm{O}(n+1)$. Since $\mathrm{O}(n+1)$ has a fixed point in $\mathbf{H}^{n+1}$, it follows from the definition that $L(\mathbf{Z})=\varnothing$. On the other hand $\Lambda_{0}=S^{n-2}$, which is the boundary of a totally geodesic submanifold $\mathbf{H}^{n-1}$ perpendicular (relative to the nondegenerate indefinite inner product) to an $\mathrm{SO}(2)$-invariant hyperbolic plane in $\mathbf{H}^{n+1}$. Furthermore, every point of an orbit $\operatorname{SO}(2) x\left(x \in S^{n}-S^{n-2}\right)$ is a cluster point of $\mathbf{Z} x$, and thus $\Lambda_{1}=S^{n}$ (= the closure of $\left.S^{n}-S^{n-2}\right), \Lambda_{2}=\varnothing$.

RemarK 2.3. Denote by $\# \Lambda$ the cardinality of components of $\Lambda$. Suppose that $\# \Lambda$ is finite. Then there exists a subgroup $\Gamma^{\prime}$ of finite index in $\Gamma$ which leaves each component invariant. If some component $\Lambda^{\prime}$ of $\Lambda$ is not a point, then $L\left(\Gamma^{\prime}\right) \subset \Lambda^{\prime}$. $\Lambda$ must be connected since $\Lambda=L(\Gamma)=L\left(\Gamma^{\prime}\right)$. When $\# \Lambda \neq 1, \Lambda$ consists of a finite number of points which are fixed by $\Gamma^{\prime}$. As remarked below Proposition 2.1, $\Gamma$ (and hence $\Gamma^{\prime}$ ) contains a nonelliptic element. And thus $\Lambda$ consists of at most two 
points; i.e., $\# \Lambda \leqslant 2$. This fact is well known in the classical theory. It is also proved by Eberlein and O'Neill for visibility manifolds. More generally, Kulkarni has shown that this fact holds for topological actions on Hausdorff spaces.

COROLlaRY 2.4. Let $\Gamma$ be a discrete subgroup of $\operatorname{Conf}\left(S^{n}\right) . \Lambda=L(\Gamma)$ consists of at most two points if and only if $\Gamma$ contains a torsion free normal solvable subgroup. In that case $\Gamma$ is conjugate to a subgroup of $E(n)$, or $\Gamma \simeq F \rtimes \mathbf{Z}$, or $\Gamma$ is an extension of $F \rtimes \mathbf{Z}$ by $\mathbf{Z}_{2}$, where $F \rtimes \mathbf{Z} \subset \mathrm{O}(n) \times \mathbf{R}^{+}$.

Proof. It is easy to see that $\Gamma$ is conjugate to a subgroup of $\operatorname{Sim}\left(\mathbf{R}^{n}\right)$, or $\Gamma$ is an extension of $\Gamma^{\prime}$ by $\mathbf{Z}_{2}$ for some $\Gamma^{\prime} \subset \operatorname{Sim}\left(\mathbf{R}^{n}\right)$. If $\Gamma \subset \operatorname{Sim}\left(\mathbf{R}^{n}\right)$, then $\Gamma \subset E(n)$ or $\Gamma \simeq F \rtimes \mathbf{Z}$ by Step 1 of Proposition 2.1. If $\Gamma$ is an extension as above, then $L\left(\Gamma^{\prime}\right)$ has exactly two fixed points of $\Gamma^{\prime}$; otherwise $\Gamma$ must be contained in $\operatorname{Sim}\left(\mathbf{R}^{n}\right)$. Thus, $\Gamma^{\prime} \simeq F \rtimes \mathbf{Z}$.

Corollary $2.5[4,12]$. Let $\Gamma$ be a discrete subgroup of $\operatorname{Conf}\left(S^{n}\right)$. Suppose that $\Omega \neq \varnothing$. Then $\Gamma$ acts properly discontinuously on $\Omega$.

Proof. Let $K$ be a compact subset of $\Omega$. It is noted that $K \cap L(\Gamma)=\varnothing$ since $\Lambda=L(\Gamma)$. It suffices to show that the set $C_{\Gamma}(K)=\{\gamma \in \Gamma \mid \gamma K \cap K \neq \varnothing\}$ is finite. Suppose that there exists a sequence $\left\{\gamma_{i}\right\} \subset \Gamma$ with $\gamma_{i} K \cap K \neq \varnothing$; by passing to a subsequence, we may assume that there are well-defined dual points $x=\lim \gamma_{i} p$, $x^{\prime}=\lim \gamma_{i}^{-1} p \in L(\Gamma)\left(p \in \mathbf{H}^{n+1}\right)$. Then for a neighborhood $U$ of $x^{\prime}$ with $U \cap K=$ $\varnothing$ and an arbitrary neighborhood $V$ of $x$, it follows that $\gamma_{n}\left(S^{n}-U\right) \subset V$. We obtain $\gamma_{n} K \subset V$, so that $K \cap V \neq \varnothing$. Therefore $x \in K$. This contradicts that $K \cap L(\Gamma)=\varnothing$.

We shall describe a relation between conformally flat manifolds and their limit sets (cf. $[10,11])$. Let $\pi \backslash X$ be a closed conformally flat manifold. Let $(d, \phi)$ be a development pair as in $\S 1$. Since $d: X \rightarrow S^{n}$ is an immersion, $d X$ is a domain. Let $\overline{d X}$ be the closure of $d X$ in $S^{n}$.

Definition 2.6. The boundary of $d X$ is defined to be the set $\overline{d X}-d X$ and is denoted by $\partial d X$. It is noted that if we put $A=S^{n}-d X$, then the set of boundary points of $A$ is $\partial d X$ and ind $\operatorname{dim} \partial d X \leqslant n-1$, where ind.dim is the inductive dimension.

It is easy to see that both $\partial d X$ and $A$ are closed subsets of $S^{n}$ which are invariant under the holonomy group $\phi(\pi)=\Gamma$. The following lemma gives a sufficient condition that $\overline{d X}=S^{n}$.

LeMma 2.7. If ind.dim $\partial d X<n-1$, then $A$ has no interior points; i.e., $\partial d X=A$.

Proof. This is an easy consequence of Alexander duality. In fact,

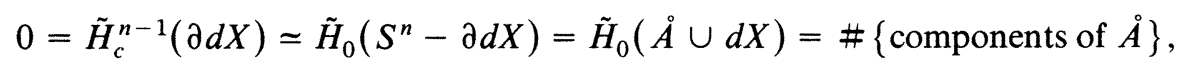

where $A$ is the set of interior points of $A$.

First we notice the following result which follows from the work of Fried [5]. 
Proposition 2.8. Let $\pi \backslash X$ be a closed conformally flat manifold. Suppose that $\partial d X=\{\mathrm{pt}\}$. Then $\pi \backslash X$ is a euclidean space form. In particular, the holonomy group $\Gamma$ is discrete and isomorphic to $\pi$. Further, $\partial d X=\Lambda=L(\Gamma)$.

Proof. If $\partial d X=\{\mathrm{pt}\}$, then $\pi \backslash X$ is uniformizable over $\mathbf{R}^{n}$ with respect to $\operatorname{Sim}\left(\mathbf{R}^{n}\right)$, where $\mathbf{R}^{n}=S^{n}-\{\mathrm{pt}\} . \pi \backslash X$ is called a similarity manifold. Then it follows from [5] that in this case $X$ is complete relative to the Riemannian flat connection, and $\pi \backslash X$ is isometric to a euclidean space form $\Gamma \backslash \mathbf{R}^{n}, \Gamma \subset E(n)$.

Proposition 2.9. Let $\pi \backslash X$ be a closed conformally flat manifold whose development map is not surjective. Suppose that the holonomy group $\Gamma$ is discrete. Then $\Gamma$ acts properly discontinuously on $d X$ and $\partial d X=\Lambda=L(\Gamma)$.

Proof. Since $d$ is not surjective, we can define $\partial d X$. We can assume that $\partial d X$ contains more than one point by Proposition 2.8. Then Proposition 2.1 and Lemma 1.3 imply that $L(\Gamma)=\Lambda \subset \partial d X$. And thus we have $d X \subset \Omega$. $\Gamma$ acts properly discontinuously on $d X$ by Corollary 2.5. If we note that $\Gamma \backslash d X$ is compact, then there exists a compact set $K$ in $d X$ for which every point of $\partial d X$ is a cluster point of $\{\gamma K\}, \gamma \in \Gamma$. It follows that $\partial d X \subset \Lambda_{2}=\Lambda$.

COROllaRY 2.10. Let $\pi \backslash X$ be a closed conformally flat manifold whose development map is not surjective. Suppose that the holonomy group $\Gamma$ is discrete. Then the development map $d: X \rightarrow d X$ is a covering map.

Proof. Let $1 \rightarrow \operatorname{Ker} \phi \rightarrow \pi \rightarrow \Gamma \rightarrow 1$ be the obvious exact sequence. Since $\Gamma$ is finitely generated, there is a torsion free normal subgroup $\Gamma^{\prime}$ of finite index in $\Gamma$. Consider the induced exact sequence $1 \rightarrow \operatorname{Ker} \phi \rightarrow \pi^{\prime} \rightarrow \Gamma^{\prime} \rightarrow 1$ associated to the above sequence. Then there is the following commutative diagram for which vertical arrows are covering maps:

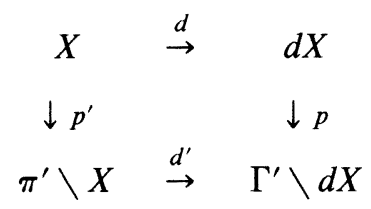

It is easy to see that $d^{\prime-1}(p(y))=\operatorname{Ker} \phi \backslash d^{-1}(y)$ for each $y \in d X$. Note that $d^{\prime}$ is a local homeomorphism as well as $d$. Thus $d^{\prime-1}(p(y))$ is discrete. Since $\Gamma^{\prime} \backslash d X$ is Hausdorff, $d^{\prime-1}(p(y))$ is closed and hence finite. Suppose that $d^{\prime-1}(p(y))$ consists of $\left\{x_{i}\right\}, i=1, \ldots, n$. Then we can choose a neighborhood $U_{i}$ of $x_{i}$ in $\pi^{\prime} \backslash X$ such that $U_{i} \cap U_{j}=\varnothing$ if $i \neq j$ and $d^{\prime}\left(U_{i}\right)$ is a neighborhood of $p(y) . V=\bigcap_{i=1}^{n} d^{\prime}\left(U_{i}\right)$ is a neighborhood of $p(y)$. Let $V^{\prime}$ be the component of $V$ containing $p(y)$. Since each component of $d^{\prime-1}\left(V^{\prime}\right)$ is mapped homeomorphically onto $V^{\prime}, d^{\prime}$ is a covering map. By the above commutativity, $d$ is also a covering map for which $d^{-1}(y) \simeq \pi_{1}(d X)$. 


\section{The discreteness property of holonomy groups.}

THEOREM 3.1. Let $M$ be an $n$ ( $\geqslant 3$ )-dimensional closed conformally flat manifold whose development map is not surjective. Let $\Gamma$ be the holonomy group. Suppose that there exists a positive dimensional closed subgroup $G$ of $\operatorname{SO}(1, n+1)$ such that $\Gamma \subset G$ and $L(\Gamma)=L(G)$. Then one of the following holds:

(i) $\Gamma$ is discrete, and $M$ is one of the following locally homogeneous Riemannian manifolds: (1) a euclidean space form, (2) a hyperbolic space form, (3) a nonnegatively curved manifold finitely covered by a Hopf manifold $S^{1} \times S^{n-1}$, (4) an $(n-k-1)$ dimensional sphere bundle over a $(k+1)$-dimensional hyperbolic space form $(1 \leqslant k \leqslant$ $n-3),(5)$ a Seifert fibered space over an $(n-1)$-dimensional hyperbolic orbifold with fiber $S^{1}$. Furthermore, a manifold of type (5) admits a Riemannian metric of nonpositive sectional curvature, and some finite covering of $M$ is diffeomorphic to the product of a circle with an $(n-1)$-dimensional hyperbolic space form.

(ii) The identity component $G^{0}$ is compact, and $G / G^{0}$ is infinite and has no torsion free normal solvable subgroups. Further, there exists a $G$-invariant $k$-dimensional sphere $S^{k}(1 \leqslant k \leqslant n-2)$ for which $L(G) \subset S^{k}$.

Proof. Let $M=\pi \backslash X$ be as before.

Step 1. Suppose that $G^{0}$ is noncompact and has compact radical. It is easy to see that $G^{0}$ has no fixed points in $S^{n}$, and $L\left(G^{0}\right)$ contains more than two points. Then it follows from [3, Lemma 4.4.5] that $G^{0}=H \cdot K$, where $H$ is a simple Lie group and $K$ is a compact Lie group. Moreover, $H$ is a transitive subgroup of a totally geodesic submanifold $\mathbf{H}^{k+1}$ in $\mathbf{H}^{n+1}(1 \leqslant k \leqslant n)$. Thus $L\left(G^{0}\right)=\partial \mathbf{H}^{k+1}=S^{k}$ $(1 \leqslant k \leqslant n)$. Since $L\left(G^{0}\right)$ is invariant under $G$, we have $L(G) \subset L\left(G^{0}\right)$. We can assume by our hypothesis and Proposition 2.8 that $\partial d X$ contains more than one point. It follows that $L(\Gamma)=L(G)=L\left(G^{0}\right)=S^{k} \subset \partial d X$ and $1 \leqslant k \leqslant n-1$. Hence, $d X \subset S^{n}-S^{k}(1 \leqslant k \leqslant n-2)$, or $d X \subset \mathbf{H}^{n}$ for a component of $S^{n}-S^{n-1}$. Choose an $\mathrm{SO}(1, k+1) \times \mathrm{O}(n-k)$-invariant metric on $S^{n}-S^{k}$ as in 1.7 of $\S 1$. The immersion $d: X \rightarrow S^{n}-S^{k}$ defines a Riemannian metric on $X$ such that $d$ is a local isometry and $\pi \subset I(X)$ with respect to that metric. Since $\pi \backslash X$ is compact, $X$ is complete. Hence, $d: X \rightarrow S^{n}-S^{k}(1 \leqslant k \leqslant n-2)$, or $d: X \rightarrow \mathbf{H}^{n}$ is a covering.

(1) Suppose $k \neq n-2$. Then $d$ is an isometry because $S^{n}-S^{k}=\mathbf{H}^{k+1} \times$ $S^{n-(k+1)}$ is simply connected. It follows that $\pi \simeq \Gamma$ is discrete, and $\pi \backslash X=$ $\Gamma \backslash \mathbf{H}^{k+1} \times S^{n-(k+1)}$ is a locally homogeneous Riemannian manifold as in 1.7 . Furthermore, for $1 \leqslant k \leqslant n-3, \pi \backslash X$ is an $(n-k-1)$-dimensional sphere bundle over a $(k+1)$-dimensional hyperbolic space form $P(\Gamma) \backslash \mathbf{H}^{k+1}$, where $P$ is the projection of $\mathrm{SO}(1, k+1) \times \mathrm{O}(n-k)$ onto the first factor. If $k=n-1, \pi \backslash X$ is a hyperbolic space form $\Gamma \backslash \mathbf{H}^{n}$.

(2) Suppose $k=n-2 . S^{n}-S^{n-2}=\mathbf{H}^{n-1} \times S^{1}$ admits a Riemannian metric of nonpositive sectional curvature, as noted in 1.7. It follows that $X=\mathbf{H}^{n-1} \times \mathbf{R}^{1}$ and $I(X)=\mathrm{SO}(1, n-1) \times E(1)$. Since $I(X)$ is transitive on $X, \pi$ is a discrete uniform subgroup of $I(X)$, and $\pi \backslash X$ is a locally homogeneous Riemannian manifold of nonpositive sectional curvature. In particular, the intersection $E(1) \cap \pi\left(=\mathbf{R}^{1} \cap \pi\right)$ 
is infinite cyclic and uniform in $\mathbf{R}^{1}$. Consider the commutative diagram

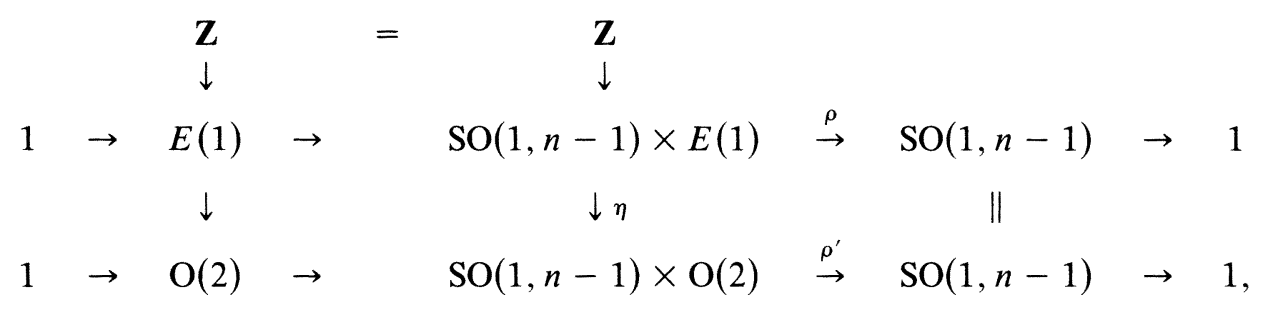

where $\left.\eta\right|_{\pi}=\phi$. Then $\rho(\pi)=\rho^{\prime}(\Gamma)$ is discrete in $\operatorname{SO}(1, n-1)$. If we put $A=E(1)$ $\cap \pi \subset \mathbf{R}^{1}$, then there is an extension $1 \rightarrow A \rightarrow \pi \rightarrow \rho(\pi) \rightarrow 1$. It is easy to see that, associated to the above extension, $\pi \backslash X$ is an injective Seifert fibered space over a hyperbolic orbifold $\rho(\pi) \backslash \mathbf{H}^{n-1}$ with typical fiber $S^{1}=A \backslash \mathbf{R}^{1}$ (exceptional fiber $S^{1}$ also) (cf. [14]). By taking a subgroup of finite index in $\pi$ if necessary, we may assume that the above extension is central. Then it follows from the results of $[7,16]$ that there is a subgroup $\pi^{\prime}$ of finite index in $\pi$ for which $\pi^{\prime} \backslash X$ is diffeomorphic (not necessarily isometric) to the product $S^{1} \times N$, where $N=\rho\left(\pi^{\prime}\right) \backslash \mathbf{H}^{n-1}$ is a hyperbolic space form. Next, in this case we prove that $\Gamma$ is discrete (this occurs if and only if $\operatorname{Ker} \phi=\pi \cap \mathbf{Z}$ is nontrivial). Suppose that $\Gamma$ were not discrete. Let $\bar{\Gamma}$ be the closure of $\Gamma$ in $\operatorname{SO}(1, n-1) \times \mathrm{O}(2)$. Then it is easy to see that $\bar{\Gamma}=\mathrm{SO}(2) \cdot \Gamma$. In particular, $\bar{\Gamma}^{0}=\mathrm{SO}(2)$. On the other hand, it follows from the property of limit sets [3, Lemma 4.3.2] that $L(\Gamma)=L(\bar{\Gamma})$. If we take $\bar{\Gamma}$ as $G$, then it satisfies the hypothesis of Theorem 3.1. Since $\bar{\Gamma}^{0}=\mathrm{SO}(2)$, this contradicts the hypothesis of Step 1 that $G^{0}$ is noncompact. As a consequence, $\pi \backslash X$ finitely covers the example $\Gamma \backslash \mathbf{H}^{n-1} \times S^{1}$ as in 1.7 (when $\Gamma$ is torsion free).

Step 2. Suppose that $G^{0}$ has a noncompact radical. It follows from the result of [8] that $G^{0}$ has a unique fixed point $\{x\}$ or exactly two fixed points $\{x, y\}$ in $S^{n}$. Since $G^{0}$ is normal in $G$, a subgroup $G^{\prime}$ of index at most 2 in $G$ has a fixed point $x$. It follows that $G^{\prime} \subset \operatorname{Sim}\left(\mathbf{R}^{n}\right)$, where $\mathbf{R}^{n}=S^{n}-\{x\}$. Now, Step 1 of Proposition 2.1 and the fact that $\operatorname{Sim}\left(\mathbf{R}^{n}\right)=\mathbf{R}^{n} \rtimes\left(\mathrm{O}(n) \times \mathbf{R}^{+}\right)$imply that $G^{\prime}$ must have the following form: $\left(\mathbf{Z}^{l} \oplus \mathbf{R}^{k}\right) \rtimes K(1 \leqslant k \leqslant n, 0 \leqslant l<n), \mathbf{R}^{k} \rtimes\left(K \times \mathbf{Z}^{+}\right)(1 \leqslant k \leqslant$ $n), K \times \mathbf{R}^{+}, \mathbf{R}^{k} \rtimes\left(K \times \mathbf{R}^{+}\right)(1 \leqslant k \leqslant n)$, where $\mathbf{Z}^{+}$is a discrete subgroup of $\mathbf{R}^{+}$ and $K$ is a compact subgroup of $\mathrm{O}(n)$. Then we obtain that $L\left(G^{\prime}\right)=\{x\}$, $L\left(G^{\prime}\right)=S^{k}(1 \leqslant k \leqslant n)$, or $L\left(G^{\prime}\right)=S^{0}$, respectively. The middle case follows since $\mathbf{R}^{k} \cup\{x\}=S^{k}$ and $\partial \mathbf{H}^{k+1}=S^{k}$ (this is called the warped product of $\mathbf{R}^{k}$ with $\mathbf{R}^{+}$; cf. [1]). It follows from the property of limit sets that $L(\Gamma)=L(G)=L\left(G^{\prime}\right)$. Note that if $L\left(G^{\prime}\right)=\{x\}$, then $G=G^{\prime}$ and $G$ is contained in the euclidean group $E(n)$. Since $L(\Gamma) \subset \partial d X$ as before, we have that $d X \subset S^{n}-S^{k}(1 \leqslant k \leqslant n), d X \subset S^{n}-$ $S^{0}$, or $d X \subset \mathbf{R}^{n}$. We prove that the first case does not occur. If $d X \subset S^{n}-S^{k}$ $(1 \leqslant k \leqslant n)$, the same argument of Step 1 shows that $\Gamma$ is discrete since $G^{0}$ is noncompact. The intersection $\Gamma^{\prime}=\Gamma \cap G^{\prime}$ has finite index in $\Gamma$. It follows from Step 1 of Proposition 2.1 that $L(\Gamma)=L\left(\Gamma^{\prime}\right)$ consists of at most two points. This contradicts the fact that $L(\Gamma)=S^{k}(1 \leqslant k \leqslant n)$. 
Choose an $\mathrm{SO}(1,1) \times \mathrm{O}(n)$-invariant metric on $S^{n}-S^{0}=\mathbf{H}^{1} \times S^{n-1}$ as in 1.7 and choose a Riemannian flat metric on $\mathbf{R}^{n}$ so that $E(n) \supset G$ is the group of isometries. Since $n \geqslant 3$, it is easy to see that $\pi \backslash X$ is a locally homogeneous Riemannian manifold $\Gamma \backslash \mathbf{H}^{1} \times S^{n-1}$ or a Riemannian flat manifold $\Gamma \backslash \mathbf{R}^{n}$, respectively. In the case that $\pi \backslash X=\Gamma \backslash \mathbf{H}^{1} \times S^{n-1}, \Gamma$ is discrete in $\operatorname{SO}(1,1) \times \mathrm{O}(n)$ and isomorphic to either $F \rtimes \mathbf{Z}$ or an extension of $F \rtimes \mathbf{Z}$ by $\mathbf{Z}_{2}$, by Corollary 2.4. Thus $\pi \backslash X$ is finitely covered by a Hopf manifold.

Step 3. From Steps 1 and 2, we can assume that $G^{0}$ is compact for any positive dimensional closed subgroups $G$ which satisfy the hypothesis of Theorem 3.1. Since $G^{0}$ is nontrivial, the set of fixed points of $G^{0}$ is a $(k+1)$-dimensional totally geodesic submanifold $\mathbf{H}^{k+1}$ in $\mathbf{H}^{n+1}$. Note that if $G^{0}$ has a unique fixed point in $\mathbf{H}^{n+1}$, then so does $G$. Thus $L(G)=\varnothing$, and $G$ is compact. It is easy to see that $M$ is a spherical space form. By our hypothesis, the development map is not surjective. Thus $0 \leqslant k \leqslant n-1$. Since $\mathbf{H}^{k+1}$ is invariant under $G$, so is $\partial \mathbf{H}^{k+1}=S^{k}$. Then $G$ sits inside the following exact sequence:

$$
\begin{array}{cllllllll}
1 & \rightarrow & A & \rightarrow & & G & \rightarrow & B & \rightarrow 1 \\
& & \downarrow & \downarrow & & & \downarrow & \\
1 & \rightarrow \mathrm{O}(n-k) & \rightarrow & \mathrm{SO}(1, k+1) \times \mathrm{O}(n-k) & \rightarrow & \mathrm{SO}(1, k+1) \rightarrow 1,
\end{array}
$$

where $A=\mathrm{O}(n-k) \cap G$ and $B=G / A$. Moreover, $A$ and $B$ satisfy the following: $1 \rightarrow G^{0} \rightarrow A \rightarrow F \rightarrow 1$ for some finite group $F, 1 \rightarrow F \rightarrow G / G^{0} \rightarrow B \rightarrow 1$. Note that $A$ leaves $S^{k}$ fixed and $B$ is discrete in $\operatorname{SO}(1, k+1)$. Since $S^{k}$ is invariant under $G$, we have $L(G) \subset S^{k}(0 \leqslant k \leqslant n-1)$. It then suffices to show that $1 \leqslant k \leqslant n-2$, and $G / G^{0}$ is infinite and has no torsion free normal solvable subgroups. First suppose that $G / G^{0}$ is finite. Then $G$ is compact. We may assume $G \subset \mathrm{O}(n+1)$. If we choose an $\mathrm{O}(n+1)$-invariant Riemannian metric of constant positive curvature on $S^{n}$, then $X$ admits a $\pi$-invariant Riemannian metric of constant positive curvature. Since $\pi \backslash X$ is compact, $X$ is complete, and thus $\pi \backslash X$ is a spherical space form $\Gamma \backslash S^{n}$. It follows that $d X=S^{n}$. This contradicts the hypothesis that $d$ is not surjective. Suppose that $G / G^{0}$ contains a torsion free normal solvable subgroup. Then so does $B$. Since $B$ is discrete in $\operatorname{SO}(1, k+1)=\operatorname{Conf}\left(S^{k}\right)$, it follows from Corollary 2.4 that $B$ leaves at most two points invariant. By taking a subgroup $G^{\prime}$ of index at most 2 in $G$, we may assume that $G^{\prime}$ has a fixed point $x$ in $S^{k}$. Thus $G^{\prime}$ is contained in $\operatorname{Sim}\left(\mathbf{R}^{n}\right)$ where $\mathbf{R}^{n}=S^{n}-\{x\}$. Then in this case $G^{\prime}$ must have the form $\mathbf{Z}^{l} \rtimes K(1 \leqslant l \leqslant k)$ or $K \times \mathbf{Z}^{+}$, where $K$ is a compact subgroup of $\mathrm{O}(n)$. We conclude that $L(G)=L\left(G^{\prime}\right)=\{x\}$ and $G \subset E(n)$, or that $L(G)=$ $L\left(G^{\prime}\right)=S^{0}$ and $G$ is either $K \times \mathbf{Z}^{+}$or $K \times\left(\mathbf{Z} \rtimes \mathbf{Z}_{2}\right)$ (cf. Corollary 2.4). In both cases, there exists a closed subgroup $\hat{G}$ of $\operatorname{SO}(1, n+1)$ with a noncompact radical for which $\Gamma \subset \hat{G}$ and $L(\Gamma)=L(\hat{G})$. This contradicts the hypothesis of Step 3. Finally, we prove that the cases $k=0, n-1$ do not occur. If $k=0$, then $B$ is a virtually abelian subgroup of $\mathrm{SO}(1,1)$. The above argument shows that this special case does not occur. If $k=n-1$, then $G^{0}=\{1\}$, which contradicts the fact that $G^{0}$ is positive dimensional. 
COROLlaRY 3.2. Let $\pi \backslash X$ be a closed conformally flat manifold for which the development map is not surjective. Suppose that the holonomy group $\Gamma$ is not discrete in Conf $\left(S^{n}\right)$. Then there exists a $\bar{\Gamma}$-invariant sphere $S^{k}(1 \leqslant k \leqslant n-2)$.

Further, if $d X \cap S^{k}=\varnothing$, then the following is true:

(1) $\pi$ is isomorphic to $\Gamma$ via the homomorphism $\phi$.

(2) $\pi \backslash X$ is a manifold of type (5).

Proof. If $\Gamma$ is not discrete, then $L(\Gamma)=L(\bar{\Gamma})$. There is a $\bar{\Gamma}$-invariant sphere $S^{k}$ $(1 \leqslant k \leqslant n-2)$ by (ii) of Theorem 3.1. If $d X \cap S^{k}=\varnothing$, then $d X \subset S^{n}-S^{k}$. As in the argument of Step 1, it is the case that $k=n-2$ and $\bar{\Gamma}^{0}=\operatorname{SO}(2)$. The result follows from the argument in the beginning of (2) of Step 1.

REMARK 3.3. There is an equivariant projection

$$
\left(\phi^{\prime}, d^{\prime}\right):(\pi, X) \rightarrow\left(\pi / A, A \backslash \mathbf{H}^{n-1} \times \mathbf{R}\right),
$$

where $A=E(1) \cap \pi \subset \mathbf{R}^{1}$. Since $A \backslash \mathbf{H}^{n-1} \times \mathbf{R}^{1}=\mathbf{H}^{n-1} \times S^{1}$ and $\phi^{\prime}(\pi) \subset$ $I\left(\mathbf{H}^{n-1} \times S^{1}\right)=\mathrm{SO}(1, n-1) \times \mathrm{O}(2)$, there is an inclusion $\left(\phi^{\prime}(\pi), \mathbf{H}^{n-1} \times S^{1}\right) \subset$ $\left(\operatorname{Conf}\left(S^{n}\right), S^{n}\right)$. Thus, $\pi \backslash X$ admits a conformally flat structure for which the holonomy group $\phi^{\prime}(\pi)$ is discrete. As a consequence, any Riemannian metric corresponding to this conformally flat structure on $\pi \backslash X$ is not conformally equivalent to the metric corresponding to the original conformally flat structure on $\pi \backslash X$.

COROLlARY 3.4. Let $M$ be an $n$ ( $\geqslant 3$ )-dimensional closed conformally flat manifold for which the development map is not surjective. Let $\Gamma$ be the holonomy group. Suppose that there exists a closed subgroup $G$ with finitely many components of $\operatorname{SO}(1, n+1)$ for which $G \supset \Gamma$ and $L(\Gamma)=L(G)$. Then $M$ is one of the locally homogeneous Riemannian manifolds in (i) of Theorem 3.1.

Note that as a consequence, the following are equivalent: Let $G$ be a closed subgroup with finitely many components in $\mathrm{SO}(1, n+1)$.

(1) $\Gamma$ is contained in $G$ and $L(\Gamma)=L(G)$.

(2) $\Gamma$ is a discrete uniform subgroup of $G$.

COROLLARY 3.5. Let $M$ be an $n(\geqslant 3)$-dimensional closed conformally flat manifold for which the development map is not surjective. Then the following are equivalent:

(1) The holonomy group $\Gamma$ belongs to $\operatorname{Sim}\left(\mathbf{R}^{n}\right)$.

(2) $\Gamma$ is virtually solvable.

In this case, $\Gamma$ is discrete, and $M$ is either a euclidean space form or a nonnegatively curved manifold finitely covered by a Hopf manifold.

Proof. $(1) \Rightarrow(2)$. If $\Gamma$ were not discrete, then $\bar{\Gamma} \subset \operatorname{Sim}\left(\mathbf{R}^{n}\right)$ and $\bar{\Gamma} / \bar{\Gamma}^{0}$ has a virtually abelian subgroup of finite index. But this does not occur, by (ii) of Theorem 3.1. Thus $\Gamma$ is discrete and, hence, virtually polycyclic.

$(2) \Rightarrow(1)$. When $\Gamma$ is virtually solvable, it is easy to see that $\Gamma$ is discrete as above. Then it follows from Corollary 2.4 that $\Gamma \subset E(n)$ or $L(\Gamma)$ consists of two points; i.e., $\Gamma \subset \mathrm{SO}(1,1) \times \mathrm{O}(n)$. Thus there exists a closed subgroup $G$ with noncompact radical for which $\Gamma \subset G$ and $L(\Gamma)=L(G)$ in each case. Hence we obtain from (i) 
of Theorem 3.1 that $M$ is a euclidean space form or finitely covered by a Hopf manifold $S^{1} \times S^{n-1}$. Consequently, the holonomy group $\Gamma$ belongs to $\operatorname{Sim}\left(\mathbf{R}^{n}\right)$.

The following is a generalization of [6] which was obtained by private discussion with W. Goldman concerning the paper of Fried [5]. Recently, I was informed that R. Schoen and S. T. Yau obtained a similar result by a different method.

THEOREM 3.6. Let $M$ be an $n$ ( $\geqslant 3$ )-dimensional closed conformally flat manifold. If the holonomy group $\Gamma$ is virtually solvable, then $M$ is a spherical space form, a euclidean space form, or a nonnegatively curved manifold finitely covered by a Hopf manifold $S^{1} \times S^{n-1}$.

Proof. Let $M=\pi \backslash X$ be as before. If $\Gamma$ is virtually solvable, then either $\Gamma$ is contained up to conjugation in $\mathrm{O}(n+1)$, or there is a subgroup $\Gamma^{\prime}$ of finite index which belongs to $\operatorname{Sim}\left(\mathbf{R}^{n}\right)$, where $\mathbf{R}^{n}=S^{n}-\left\{x_{0}\right\}$ for some point $x_{0}$ (cf. [6, Lemma 1.4]). If $\Gamma \subset \mathrm{O}(n+1)$, it is easy to see that $\pi \backslash X$ is a spherical space form. Suppose that $\Gamma^{\prime} \subset \operatorname{Sim}\left(\mathbf{R}^{n}\right)$. Put $X^{\prime}=X-d^{-1}\left(x_{0}\right)$ and $\pi^{\prime}=\phi^{-1}\left(\Gamma^{\prime}\right)$. Then

$$
\pi^{\prime} \backslash X^{\prime}=\pi^{\prime} \backslash X-F
$$

where $F=\pi^{\prime} \backslash d^{-1}\left(x_{0}\right)$ consists of finite points. $\pi^{\prime} \backslash X^{\prime}$ is a similarity manifold with the same development map $d: X^{\prime} \rightarrow \mathbf{R}^{n}$.

Case 1. Suppose that $X^{\prime}$ is complete (relative to the Riemannian flat metric). Then $d: X^{\prime} \rightarrow \mathbf{R}^{n}$ is an isometry. Thus $\Gamma^{\prime}\left(\simeq \pi^{\prime}\right)$ is discrete in $\operatorname{Sim}\left(\mathbf{R}^{n}\right)$. It follows that either $\Gamma^{\prime} \subset E(n)$ or $\Gamma^{\prime} \simeq \mathbf{Z}$ by Step 1 of Proposition 2.1. The latter case does not occur since $\Gamma^{\prime}$ has exactly two fixed points. If $\operatorname{rank} \Gamma^{\prime}=k>0$ for $\Gamma^{\prime} \subset E(n)$, then there is a totally geodesic compact submanifold $\Gamma^{\prime} \backslash \mathbf{R}^{k}$ in $\Gamma^{\prime} \backslash \mathbf{R}^{n}$ (cf. [21]). Thus, $\pi^{\prime} \backslash d^{-1}\left(\mathbf{R}^{k}\right)$ is a closed submanifold of $\pi^{\prime} \backslash X$. It implies that $d^{-1}\left(\mathbf{R}^{k}\right)=X$. Hence $d^{-1}\left(x_{0}\right)=\varnothing$.

Case 2. Suppose $X^{\prime}$ is incomplete. Then an argument similar to one in [5, p. 580] shows that there exists a certain nonempty set outside $d X^{\prime}$. Thus, in both cases, $d$ : $X \rightarrow S^{n}$ is not surjective. The result follows from Corollary 3.5.

We are now ready to prove the main theorem.

THEOREM 3.7. Let $M$ be a closed conformally flat 3-manifold for which the development map is not surjective. Then one of the following is true:

(1) The holonomy group $\Gamma$ is discrete.

(2) $M$ is a locally homogeneous Riemannian manifold of nonpositive sectional curvature. In this case, some finite covering of $M$ is diffeomorphic to the product of a circle with a compact surface of genus $g(\geqslant 2)$.

Further, in each case the development map is a covering map.

Proof. Suppose that $\Gamma$ is not discrete in $\operatorname{Conf}\left(S^{3}\right)$. Then by (ii) of Theorem 3.1, the group $G=\bar{\Gamma}$ sits inside the exact sequence (cf. Step 3)

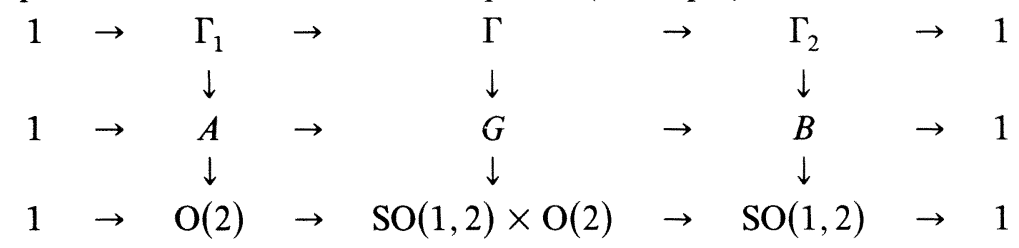


where $\Gamma_{1}=A \cap \Gamma$ is a nontrivial subgroup of $\mathrm{O}(2)$. Moreover, we note that $\mathrm{O}(2)$ leaves $S^{1}$ fixed, where $S^{1}=\partial \mathbf{H}^{2}$ and $B$ is discrete in $\operatorname{SO}(1,2)$. Since $\Gamma_{2}$ is a finitely generated discrete subgroup of $\mathrm{SO}(1,2)$, passing to a subgroup of finite index, it is either a free group of finite rank $(\geqslant 2)$ or isomorphic to the fundamental group of a compact surface of genus $g(\geqslant 2)$. Let $M=\pi \backslash X$ be as before. If we prove that $d X \cap S^{1}=\varnothing$, then the result follows from Corollary 3.2.

Case 1. Suppose that $\Gamma_{2}$ is uniform in $\operatorname{SO}(1,2)$. It is easy to see that $L(\Gamma)=$ $L\left(\Gamma_{2}\right)=L(\operatorname{SO}(1,2))=S^{1}$. Since we can assume $L(\Gamma) \subset \partial d X$ as before, we obtain $d X \cap S^{1}=\varnothing$. In this case, we know that $d: X \rightarrow \mathbf{H}^{2} \times S^{1}$ is covering. (See Step 1 of Theorem 3.1.)

Case 2. Suppose that $\Gamma_{2}$ is a free group. We will see that this case does not occur. $L(\Gamma)=L\left(\Gamma_{2}\right)$ is a totally disconnected perfect set lying in $S^{1} \cap \partial d X$. By taking a subgroup of finite index in $\Gamma$ if necessary, we may assume that $\Gamma_{1} \subset \mathrm{SO}(2)$ and that the exact sequence $1 \rightarrow \Gamma_{1} \rightarrow \Gamma \rightarrow \Gamma_{2} \rightarrow 1$ is a central extension. (Note that every subgroup of finite index in $\Gamma$ is finitely generated since $\pi$ is.) Since $\Gamma_{2}$ is a free group of finite rank $(\geqslant 2)$, this extension splits; i.e., $\Gamma=\Gamma_{1} \times \Gamma_{2}^{\prime}$, where $\Gamma_{2}^{\prime}$ is isomorphic to $\Gamma_{2}$. Thus, $\Gamma_{2}^{\prime}$ is discrete in $\operatorname{SO}(1,2) \times \operatorname{SO}(2)$, and $L\left(\Gamma_{2}^{\prime}\right)=L\left(\Gamma_{2}\right)$. If we set $\Omega=S^{3}-L\left(\Gamma_{2}^{\prime}\right)$, then it follows from Proposition 2.1 and Corollary 2.5 that $\Gamma_{2}^{\prime}$ acts properly discontinuously (and hence freely) on $\Omega$. Since $\operatorname{SO}(2)$ leaves $L\left(\Gamma_{2}^{\prime}\right)$ $\left(\subset S^{1}\right)$ fixed, the set $L\left(\Gamma_{2}^{\prime}\right)$ is invariant under the group $\mathrm{SO}(2) \cdot \Gamma$. We then have an induced action of $\mathrm{SO}(2)$ on $\Gamma_{2}^{\prime} \backslash \Omega$, where it is noted that $\Gamma_{2}^{\prime}$ is normal in $\mathrm{SO}(2) \cdot \Gamma$ and $\mathrm{SO}(2)=\mathrm{SO}(2) \cdot \Gamma / \Gamma_{2}^{\prime}$. Choose an $\mathrm{SO}(2)$-invariant Riemannian metric on $\Gamma_{2}^{\prime} \backslash \Omega$, and let $g$ be its lift of the metric on $\Omega$.

Now, suppose that $d X \cap S^{1} \neq \varnothing$. Since $d X \subset \Omega$, we see that $\operatorname{SO}(2)$ has a fixed point in $\Omega$. Then the group of all liftings of $\mathrm{SO}(2)$ to isometries (relative to $g$ ) of $\Omega$ contains the group $\mathrm{SO}(2) \cdot \Gamma$. (For example, see [25].) The immersion $d: X \rightarrow \Omega$ defines a $\pi$-invariant Riemannian metric on $X$ so that $d$ is a local isometry. Since $X$ is complete and $\Omega$ is simply connected, the map $d: X \rightarrow \Omega$ is an isometry. This contradicts the fact that $\Gamma$ is indiscrete, and hence $d X \cap S^{1}=\varnothing$. Moreover, it follows from Corollary 3.2 that $\Gamma_{2}$ is isomorphic to the fundamental group of a compact surface of genus $g(\geqslant 2)$. As a consequence, Case 2 cannot occur. This completes the proof of (2).

When $\Gamma$ is discrete, the immersion $d: X \rightarrow d X$ is a covering by Corollary 2.10 .

Note. The above conformally flat manifold $\Gamma_{2}^{\prime} \backslash \Omega$ is the connected sum of a finite number of copies of Hopf manifolds $S^{1} \times S^{2}$. This follows from the fact that $\Gamma_{2}^{\prime}$ is a finitely generated Schottky-Fuchsian group (i.e., $\Gamma_{2}^{\prime}$ is a free group of rank $k$ $(2 \leqslant k<\infty)$ in $\operatorname{PSL}(2, \mathbf{R}))$.

Problem 1. Let $\Gamma$ be a torsion free discrete subgroup of $\operatorname{Conf}\left(S^{3}\right)$. If $\Lambda=L(\Gamma)$ is a totally disconnected perfect set, then is $\Gamma \backslash \Omega$ the connected sum of a finite number of copies of euclidean space forms and Hopf manifolds?

Problem 2. Let $M^{3}$ be a closed conformally flat manifold whose development map is not surjective. Suppose that the boundary $C$ of the development image is a Jordan curve. Then

(1) If $C$ is rectifiable, is $M$ a Seifert fibered space of type (5) of Theorem 3.1? 
(2) If $C$ is nonrectifiable, must such a conformally flat manifold be a compact surface of genus $g(\geqslant 2) \times S^{1}$ ?

It is conceivable that Kulkarni's result [12, Theorem 6.11] gives an affirmative answer to Problem 1. In Problem 2, it is noted that the holonomy group $\Gamma$ is discrete and $L(\Gamma)=C$. If one can prove that $\Gamma$ is Fuchsian when $C$ is rectifiable (and thus $C=S^{1}$ ), then (1) is true (cf. [24]). For (2), by making use of quasi-Fuchsian groups, it is easy to see that the product $M_{g} \times S^{1}$ (where $M_{g}$ is a compact surface of genus $g \geqslant 2$ ) admits a conformally flat structure for which the boundary is a nonrectifiable quasi-circle (cf. [28]).

COROLlARY 3.8. Let $M$ be an $n$ ( $\geqslant 3$-dimensional closed conformally flat manifold. If the development map is not surjective, then it is a covering map.

Proof. If $\Gamma$ is not discrete, then as in the proof of Theorem 3.7, $\Gamma$ sits in the following exact sequence:

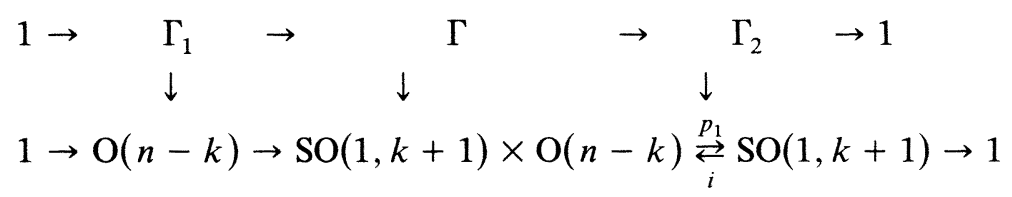

Note that $\mathrm{O}(n-k)$ leaves $S^{k}$ fixed (cf. Step 3). Then $\Gamma$ is contained in the group $i\left(\Gamma_{2}\right) \times \mathrm{O}(n-k)$ and $L\left(i\left(\Gamma_{2}\right)\right)=L\left(\Gamma_{2}\right)=L(\Gamma) \subset \partial d X \cap S^{k} \quad(1 \leqslant k \leqslant n-2)$. Moreover, $i\left(\Gamma_{2}\right)$ is discrete and it acts properly discontinuously on $\Omega=S^{n}-$ $L\left(i\left(\Gamma_{2}\right)\right)$. By taking a subgroup of finite index in $\Gamma$, we can assume that $i\left(\Gamma_{2}\right)$ acts freely on $\Omega$. Now, if $d X \cap S^{k} \neq \varnothing$, then it follows from the same argument of Theorem 3.7 that $X$ admits a $\pi$-invariant Riemannian metric for which $d: X \rightarrow \Omega$ is a local isometry. Since $\pi \backslash X$ is compact, $X$ is complete, and hence $d: X \rightarrow d X=\Omega$ is a covering.

\section{REFERENCES}

1. R. Bishop and B. O'Neill, Manifolds of negative curvature, Trans. Amer. Math. Soc. 145 (1969), $1-44$.

2. S. Chen and P. Eberlein, Isometry groups of simply connected manifolds of nonpositive curvature, Illinois J. Math. 74 (1980), 73-103.

3. S. Chen and L. Greenberg, Hyperbolic spaces, Contribution to Analysis, Academic Press, New York, 1974, pp. 49-87.

4. P. Eberlein and B. O’Neill, Visibility manifolds, Pacific J. Math. 46 (1973), 45-109.

5. D. Fried, Closed similarity manifolds, Comment. Math. Helv. 55 (1980), 576-582.

6. W. M. Goldman, Conformally flat manifolds with nilpotent holonomy and the uniformization problem for 3-manifolds, Trans. Amer. Math. Soc. 278 (1983), 573-583.

7. D. Gromoll and J. Wolf, Some relations between the metric structure and the algebraic structure of the fundamental group in manifolds of nonpositive"curvature, Bull. Amer. Math. Soc. 77 (1971), 545-552.

8. Y. Kamishima, Lorentz space forms and virtually solvable groups, Indiana Univ. Math. J. 34 (1985), 249-257.

9. S. Kobayashi, Transformation groups in differential geometry, Springer-Verlag, New York, 1972.

10. N. Kuiper, On conformally flat manifolds in the large, Ann. of Math. (2) 50 (1949), 916-924.

11. , On compact conformally euclidean spaces of dimension > 2, Ann. of Math. (2) 52 (1950), $478-490$.

12. R. S. Kulkarni, Groups with domains of discontinuity, Math. Ann. 237 (1978), 253-272.

13. On the principle of uniformizations, J. Differential Geom. 13 (1978), 109-138. 
14. R. S. Kulkarni and F. Raymond, Three-dimensional Lorentz space forms, J. Differential Geom. (to appear).

15. A. G. Kurosh, Theory of groups, Vols. I, II, Chelsea, New York, 1960.

16. H. B. Lawson and S. T. Yau, Compact manifolds of nonpositive curvature, J. Differential Geom. 7 (1972), 211-228.

17. M. L. Mihalik, Ends of double extension groups, Preprint, 1984.

18. P. Scott, The geometries of 3-manifolds, Bull. London Math. Soc. 15 (1983), 401-487.

19. __ There are no fake Seifert fiber spaces with infinite $\pi_{1}$, Ann. of Math. (2) 117 (1983), 35-70.

20. J. Stallings, Group theory and 3-dimensional manifolds, Yale Math. Monographs 4, Yale Univ. Press, 1971.

21. J. Wolf, Spaces of constant curvature, McGraw-Hill, New York, 1967.

22. H. Takagi, Conformally flat Riemannian manifolds admitting a transitive group of isometries, Tôhoku Math. J. 27 (1975), 103-110.

23. D. Sullivan, The density at infinity of a discrete group of hyperbolic motions, Inst. Hautes Études Sci. Publ. Math. 50 (1979), 419-450.

24. L. Bers, Uniformization, moduli and Kleinian groups, Bull. London Math. Soc. 4 (1972), 257-300.

25. G. Bredon, Introduction to compact transformation groups, Academic Press, New York, 1972.

26. W. M. Goldman, Projective structures and Fuchsian holonomy, preprint, 1985.

27. W. Thurston, The geometry and topology of 3-manifolds, Princeton Univ. Press, Princeton, N.J., 1979.

28. Y. Kamishima, Conformal transformations and closed conformally flat 3-manifolds. II, preprint, 1985.

Department of Mathematics, University of OKlahoma, Norman, OKlahoma 73019

Current address: Department of Mathematics, Hokkaido University, Sapporo, 060 Japan. 